\title{
Longitudinal adherence to antiretroviral drugs for preventing mother-to-child transmission of HIV in Zambia
}

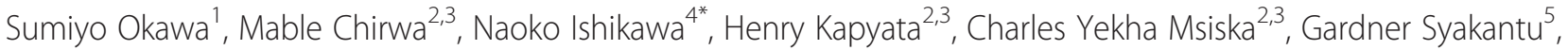
Shinsuke Miyano ${ }^{4}$, Kenichi Komada, ${ }^{3,4}$, Masamine Jimba' and Junko Yasuoka ${ }^{1}$

\begin{abstract}
Background: Adherence to antiretroviral (ARV) drugs is essential for eliminating new pediatric infections of human immunodeficiency virus (HIV). Since the Zambian government revised the national guidelines based on option A (i.e., maternal zidovudine and infant ARV prophylaxis) of the World Health Organization's 2010 guidelines, no studies have assessed adherence to ARVs during pregnancy up to the postpartum period. This study aimed to examine adherence to ARVs and identify the associated risk factors.

Methods: A prospective cohort study was conducted in the Chongwe district from June 2011 to January 2014. Self-reported adherence to ARVs was examined during pregnancy and at one week, six weeks, and 24 weeks postpartum among 321 HIV-positive women. The probability of remaining adherent to ARVs was estimated using the Kaplan-Meier method, and the risk factors for non-adherence were identified using the Cox proportional hazard regressions - treating loss to follow-up as non-adherence. The statuses of HIV in HIV-exposed infants were assessed in January 2014.
\end{abstract}

Results: During the study period, 326 infants were born to HIV-positive women, 262 (80.4\%) underwent HIV testing, and 11 (3.4\%) had their HIV infection detected at the time that they had the latest HIV testing as of January 2014. The ARV adherence rate was $82.5 \%$ during pregnancy, $84.2 \%$ at one week postpartum, $81.5 \%$ at six weeks postpartum, and $70.5 \%$ at 24 weeks postpartum. The probability of remaining adherent to ARVs was 0.61 at day 50, 0.35 at day 100, 0.18 at day 200, and 0.06 at day 300 . Attending a referral health center (HC) was a risk factor for non-adherence compared with attending rural HCs that provided HIV care/treatment (adjusted hazard ratio [aHR] 0.71, $95 \%$ confidence interval [CI] 0.57-0.88) and those that did not provide HIV care/treatment (aHR $0.58,95 \% \mathrm{Cl}$ 0.46-0.74). A new diagnosis of HIV infection compared to a known HIV-positive status before pregnancy was another risk factor for non-adherence (aHR 1.24, $95 \% \mathrm{Cl} 1.03-1.50$ ).

Conclusions: Maintaining adherence to ARVs through pregnancy to the postpartum period remains a crucial challenge in Zambia. To maximize the treatment benefits, adherence to ARVs and retention in care should be improved at all health facilities.

Keywords: Prevention of mother-to-child transmission of HIV (PMTCT), Antiretroviral (ARV), Adherence, Pregnancy, Zambia

\footnotetext{
* Correspondence: n-ishikawa@it.ncgm.go.jp

${ }^{4}$ National Center for Global Health and Medicine, 1-21-1 Toyama,

Shinjuku-ku, Tokyo 162-8655, Japan

Full list of author information is available at the end of the article
} 


\section{Background}

The global epidemic of human immunodeficiency virus (HIV) has affected the lives of millions of children. In 2013, about 240,000 children were newly infected with HIV, with the majority of pediatric HIV infections being attributed to mother-to-child transmission [1]. In an attempt to eliminate new pediatric HIV infections, the World Health Organization (WHO) revised the guidelines for preventing mother-to-child transmission of HIV (PMTCT) in 2010 [2].

The guidelines recommended two options for antiretroviral (ARV) prophylaxis. In option A, pregnant women begin zidovudine (AZT) at 14 gestational weeks and continue it during pregnancy, while their infants receive nevirapine (NVP) throughout the breastfeeding period. In option $\mathrm{B}$, pregnant women receive triple ARV prophylaxis from 14 weeks of pregnancy to the end of the breastfeeding period, while their infants receive NVP for the first four to six weeks. In both options, lifelong antiretroviral therapy (ART) is recommended for all women with a CD4 cell count $\leq 350$ cells $/ \mathrm{mm}^{3}$ or those in the WHO clinical stage 3 or 4. It is expected that ARV prophylaxis can reduce the risk of HIV transmission from 35 to $5 \%$ among breastfeeding infants [2]. In 2013, WHO released new consolidated guidelines on the use of ARVs, in which two options for PMTCT, option B and B+ were recommended [3]. In option $\mathrm{B}+$, all women initiate lifelong triple $\mathrm{ARVs}$ regardless of their WHO clinical stage or CD4 cell count [3].

Optimal adherence to ARVs is essential to protect children from acquiring HIV, maintain maternal health, and minimize the risk of drug resistance [4]. According to a meta-analysis on ARV adherence in the PMTCT program, $74 \%$ of pregnant women in low-, middle-, and high-income countries achieved adequate adherence, and the ARV adherence rate was higher during pregnancy than the postpartum period [5].

In sub-Saharan Africa, only a few studies have assessed ARV adherence during pregnancy and the postpartum period [6-13]. In these studies, disclosure of the HIV status was a major factor that affected adherence among pregnant women $[6-9,13]$. Other risk factors for non-adherence included a younger maternal age, lack of income-generating activity, early enrollment in the PMTCT services, experiences of discrimination, and lack of treatment support $[6,7,9,13]$.

ARV adherence is affected by the loss of patient follow-up [14, 15], and a loss to follow-up has been commonly observed in the PMTCT program in subSaharan Africa [16-18]. The effects of loss to followup on ARV adherence will become more serious in the longitudinal ARV regimen newly introduced in the WHO's 2010 guidelines. However, few studies have examined longitudinal ARV adherence and the effects of the loss to follow-up.
Zambia is one of the countries seriously affected by HIV, with an estimated HIV prevalence of $12.6 \%$ among the adult population in 2013 [19]. The national guidelines for PMTCT were revised based on option A of the WHO 2010 guidelines, which were implemented across the country at the time that the present study was conducted [20]. In accordance with the WHO 2013 guidelines, the Zambian government has been shifting to option $\mathrm{B}+$, which is gradually being introduced at qualified heath facilities. In 2011, the majority of pregnant women (97\%) attended an antenatal clinic and underwent HIV testing, and $85 \%$ of HIV-positive pregnant women received ARVs; however, only $36 \%$ of infants born to HIV-positive women received ARVs [19]. The mother-to-child transmission rate was $12 \%$ in 2012 [19]. However, the availability of HIV care/treatment services is limited. In 2010, there were 1784 health facilities in the country, and only 453 (25.4\%) offered HIV care/ treatment services [21]. By 2012, about 110 facilities introduced HIV care/treatment services [19].

Before introducing the WHO 2010 guidelines, several studies have examined the uptake of ARVs among HIVpositive women and their infants in Zambia [22-28]. According to these studies, the maternal factors associated with a missed ARV dose were a longer interval between HIV testing and delivery [23], no high school-level education, lower newborn birth weight [24], maternal age between 26 and 30 years, multi-gravidity, fewer antenatal clinic visits, vaginal delivery, single-dose NVP regimen versus ART regimen [28], illiteracy, and no history of prior fetal or infant death [22]. In addition, the first diagnosis of HIV infection during pregnancy is a crucial factor that affects ARV adherence [29]. According to the new guidelines, ARV adherence and ART initiation can be affected by the availability of HIV care/treatment services, wherein PMTCT services are provided [30]. However, few studies have focused on these factors that affect ARV adherence in the PMTCT program in Zambia.

To date, no studies have examined the long-term ARV adherence under the 2010 national guidelines in Zambia. An evaluation of longitudinal ARV adherence would provide a platform to examine the feasibility of the WHO 2013 guidelines. This study aimed to assess ARV adherence from pregnancy to 24 weeks postpartum by considering the effect of loss to follow-up, and identify risk factors for non-adherence to ARVs.

\section{Methods}

Study setting

This prospective cohort study was conducted in Chongwe district, Zambia from June 2011 to January 2014. At the beginning of the study, there were 39 health facilities across the district. This study included the western part of the district as the study site where the 
Chongwe referral health center (HC) and 19 rural HCs are operated. All of the facilities have implemented the PMTCT program under the 2010 national guidelines, and only six provided HIV care/treatment. The Chongwe referral $\mathrm{HC}$ was equivalent to a district hospital, and it supervised the 19 rural $\mathrm{HCs}$ with regards to the implementation of the PMTCT and HIV care/treatment programs.

Of these health facilities, 11 were selected as the study sites. First, we selected all facilities that offered PMTCT services and HIV care/treatment, including one referral $\mathrm{HC}$ and five rural HCs. The HIV care/treatment was provided daily at the Chongwe referral $\mathrm{HC}$ and fortnightly at the five rural HCs. Then of 14 rural HCs, we selected five rural HCs that did not provide HIV care/treatment. At these facilities, women eligible for ART were referred to the Chongwe referral $\mathrm{HC}$ or one of the five rural $\mathrm{HCs}$ that provided HIV care/treatment. The five rural HCs with no HIV care/treatment were selected based on the following inclusion criteria: located within $20 \mathrm{~km}$ from a neighboring health facility offering HIV care/treatment, and delivery care was provided. Finally, 11 health facilities were included in the study.

\section{Data collection}

HIV-positive women were recruited by nurses or trained volunteers when they attended PMTCT services from pregnancy (median 10 weeks before delivery) to the postpartum period (median six weeks after delivery). During the study period, we enrolled 360 women in the study, and analyzed 321 eligible women. Then, 39 women were excluded for the following reasons: 29 missed key data; eight had an abortion or stillbirth during pregnancy; and two were not breastfeeding at recruitment.

During the study period, participants had a maximum of four interviews at the following periods: during pregnancy (first observation), one week postpartum (second observation), six weeks postpartum (third observation), and 24 weeks postpartum (forth observation). The cutoff points for the follow-up observations were set at five weeks postpartum for the second observation, 23 weeks postpartum for the third observation, and 52 weeks postpartum for the fourth observation. All observations were completed either at the fourth observation, at the end of the interview survey (October 1, 2012), or at 52 weeks postpartum. The time points for the postpartum observations were decided by considering the timing of routine PMTCT visits [20].

Structured questionnaires were developed based on standardized questionnaires produced by the WHO [31], 2007 Zambia Demographic and Health Survey [32], and Adult AIDS Clinical Trials Group [33, 34]. Through face-to-face interviews, participants provided their age, educational level, marital status, type of health facility (referral HC, rural HCs with HIV care/treatment, or rural HCs without HIV care/treatment), travel time from home to the health facility, HIV status at enrollment in the PMTCT program, timing of study enrollment (pregnancy or postpartum), ARV regimen (ARV prophylaxis or ART), breastfeeding status, internalized stigma (i.e., feeling bad about being HIV-positive), and attitude about taking ARVs with three items (taking ARVs can prevent HIV transmission to the baby; taking ARVs makes me healthier; and if I do not take ARVs properly, the ARVs will not work). Agreement with the three questions was regarded as having a positive attitude towards ARVs.

As for the partners' characteristics, participants provided their experience with couple HIV testing and counseling (CHTC), disclosure of their HIV status to their partner, HIV status of the partner, and experience with domestic violence (e.g., verbal abuse, physical abuse, and/or being forced to leave home) [35]. If a woman reported any of the three types of domestic violence, it was defined as having experienced domestic violence.

Using the PMTCT-related register, we assessed the HIV status and survival status of infants who were born to the study participants as of January 2014. During the study period, these infants were recommended to undergo HIV testing at six weeks, six months, 12 months, and 18 months of age at the study sites. We examined each date of HIV testing that the infants had ever undergone. If an infant had undergone HIV testing more than once, their latest HIV status was used in the analysis. However, we were not able to confirm the breastfeeding status at the time that the infants underwent HIV testing because a number of information was missing in the register.

\section{Definition of the study outcome}

This study examined adherence to ARVs as the primary outcome. To measure adherence to ARVs, we used the self-report questionnaire developed by Adult AIDS Clinical Trials Group [36]. Despite the potential risk of overestimation, the self-report measurements have been validated [37], and they are widely used in resourcelimited settings [5]. During the study period, we used the national PMTCT guidelines based on the option A of WHO 2010 guidelines [20]. Eleven HCs prescribed AZT for women not eligible for treatment during pregnancy and NVP syrup for HIV-exposed infants during the postpartum period under the PMTCT program, whereas triple ARV drugs for women on ART were prescribed from pregnancy to the postpartum period under the HIV care/treatment service program at the Chongwe referral $\mathrm{HC}$ or at the five rural HCs offering HIV care/ treatment.

Corresponding to the national protocol, ARV adherence was measured differently between women on ARV 
prophylaxis and those on ART. For women on ARV prophylaxis, maternal adherence was assessed during pregnancy, and infant adherence was assessed during the postpartum period. For those on ART, maternal adherence was measured over the assessment period.

For both groups of women, non-adherence was defined as having missed any prescribed drug or having failed to follow the prescribing schedule at least once in the four days preceding the interview $[7,38]$. Medication interruption was also defined as non-adherence if it was detected during the interview (primary definition).

For the women on ARV prophylaxis, the assessment of ARV adherence was completed on cessation of breastfeeding and confirmation of the HIV-negative status of their infants, as there was no longer a risk of HIV transmission to their infants. However, women on ART continued the assessment of ARV adherence regardless of the breastfeeding status, as they require lifelong ART.

Additionally, the authors expected that a number of participants would be lost to follow-up and would likely not adhere to ARVs during the period of loss to followup [15]. To include participants lost to follow-up in the analysis, we adopted a broader definition of nonadherence in which delayed or missed scheduled visits and reported infant deaths were treated as nonadherence (secondary definition).

\section{Statistical analysis}

In the descriptive analysis, the chi-square test was performed to assess proportional differences in the categorical variables. The ARV adherence rate was calculated at four observed periods among the women who had attended the scheduled interviews, which did not include the women who had missed scheduled visits (primary definition).

The probability of remaining adherent to ARVs was estimated using the Kaplan-Meier method, which applies the secondary definition of ARV adherence using the time from the start of an observation to the detection of nonadherence to ARVs or interruption of ARV administration due to loss to follow-up (an event). However, study participants may repeat the events during the assessment period, and the observed record could contain intervalcensored records. To analyze multiple events, the recurrent event model was used in the analysis [39]. In this model, the censorship point adopted the midpoint of the previous observation and the current non-adherence events (the main model), as a non-adherent event was likely to have occurred before it was detected [40]. Observations were also censored at the midpoint between the previous observation and the cutoff point of the present observation if a participant was lost to follow-up. Changes in the ARV regimen from ARV prophylaxis to ART were treated as a time-dependent variable.
The proportional hazards assumption was tested using a log-log survival plot and Schoenfeld residuals. The Cox proportional hazard regression model was subsequently tested to identify the risk factors for non-adherence to ARVs in a backward stepwise model-building procedure. The final model-building process assigned independent variables to two groups: those regarded as essential in the study (i.e., age, educational level, marital status, health facility type, and timing of HIV diagnosis) were retained regardless of their significance, and other potential confounders were retained if the $p$-value was $<0.05$. In the Cox proportional hazard models, the potential correlations within individuals were adjusted using robust variance estimates.

Sensitivity analyses were performed to test the effect of the time definitions and ARV regimen on the study outcome. Regarding the effect of the time definition, an alternative model was developed in which crude cutoff points were applied to censorship to estimate the time to detect a non-adherent event. Subsequently, we assessed any differences in the hazard ratios between censorship at the midpoint (the main model) and at the crude cutoff point (the alternative model). Similarly, adherence to ARVs among those on ARV prophylaxis and those on ART were analyzed separately to examine any differences in the hazard ratios between the two ARV regimens.

The statistical analysis was performed using Stata SE, version 12 (Stata Corp., College Station, TX).

\section{Ethical considerations}

This study was approved by the Institutional Ethics Committee of the National Center for Global Health and Medicine, the Research Ethics Committee of the University of Tokyo, and Biomedical Research Ethics Committee of the University of Zambia. Written informed consent was obtained from all participants at recruitment. When a participant was found to have any physical or psychosocial problems (e.g., domestic violence) through the interview, she was referred to health workers for further follow-up.

\section{Results}

\section{Basic characteristics of the study participants}

Table 1 shows the basic characteristics of the 321 HIVpositive women. The referral HC enrolled 130 women (40.5\%); the five rural HCs with HIV care/treatment enrolled 106 (33.0\%) women; and the five rural HCs without HIV care/treatment enrolled 85 women (26.5\%). Half of the women (49.8\%) were recruited in the study during pregnancy. The median age was 29 years (interquartile range [IQR] 24-34), 105 (32.7 \%) had achieved an educational level higher than primary school (eight years), and $272(84.7 \%)$ were married or living with a partner. The median time required to access the nearest 
Table 1 Basic characteristics of study participants

\begin{tabular}{|c|c|c|c|c|c|}
\hline Characteristics & Overall & Referral $\mathrm{HC}^{\mathrm{a}}$ & Rural HCs with HIV care/treatment & Rural HCs without HIV care/treatment & $p$-value ${ }^{\ddagger}$ \\
\hline$N(\%)$ & 321 & $130(40.5)$ & $106(33.0)$ & $85(26.5)$ & \\
\hline \multicolumn{6}{|l|}{ Age } \\
\hline Median (IQR $\left.{ }^{\mathrm{b}}\right)$ & $29(24-34)$ & $30(24-33)$ & $30.5(26-34)$ & $28(22-35)$ & \\
\hline$\leq 29$ & $163(50.8)$ & $64(49.2)$ & $48(45.3)$ & $51(60.0)$ & \multirow[t]{2}{*}{0.1} \\
\hline$\geq 30$ & $158(49.2)$ & $66(50.8)$ & $58(54.7)$ & $34(40.0)$ & \\
\hline \multicolumn{6}{|l|}{ Educational level } \\
\hline$\leq$ Primary & $216(67.3)$ & $76(58.5)$ & $81(76.4)$ & $59(69.4)$ & \multirow[t]{2}{*}{0.01} \\
\hline$\geq$ Secondary & $105(32.7)$ & $54(41.5)$ & $25(23.6)$ & $26(30.6)$ & \\
\hline \multicolumn{6}{|l|}{ Marital status } \\
\hline Married/living with partner & $272(84.7)$ & $112(86.2)$ & 95 (89.6) & $65(76.5)$ & \multirow[t]{2}{*}{0.04} \\
\hline Other & $49(15.3)$ & $18(13.9)$ & $11(10.4)$ & $20(23.5)$ & \\
\hline \multicolumn{6}{|l|}{ Travel time to health facility } \\
\hline Median (IQR $)$ & $60(30-90)$ & $30(20-60)$ & $90(60-120)$ & $60(35-120)$ & \\
\hline$\leq 59 \min$ & $134(41.7)$ & $84(64.6)$ & $21(19.8)$ & $29(34.1)$ & \multirow[t]{2}{*}{$<0.01$} \\
\hline$\geq 60 \min$ & $187(58.3)$ & $46(35.4)$ & $85(80.2)$ & $56(65.9)$ & \\
\hline \multicolumn{6}{|l|}{ HIV status at PMTCT enrolment } \\
\hline Already known HIV status & $162(50.5)$ & $68(52.3)$ & $68(64.2)$ & $26(30.6)$ & \multirow[t]{2}{*}{$<0.01$} \\
\hline Newly diagnosed & $159(49.5)$ & $62(47.7)$ & $38(35.9)$ & $59(69.4)$ & \\
\hline \multicolumn{6}{|l|}{ ARV regimen at study enrolment } \\
\hline ARV prophylaxis & $167(52.0)$ & $66(50.8)$ & $49(46.2)$ & $52(61.2)$ & \multirow[t]{2}{*}{0.1} \\
\hline ART & $154(48.0)$ & $64(49.2)$ & $57(53.8)$ & $33(38.8)$ & \\
\hline \multicolumn{6}{|l|}{ Timing of study enrolment } \\
\hline During pregnancy & $160(49.8)$ & $55(42.3)$ & $62(58.5)$ & $43(50.6)$ & \multirow[t]{2}{*}{0.05} \\
\hline After delivery & $161(50.2)$ & $75(57.7)$ & $44(41.5)$ & $42(49.4)$ & \\
\hline \multicolumn{6}{|l|}{ Baseline ARV adherence } \\
\hline Adherent & $254(79.1)$ & $102(78.5)$ & $89(84.0)$ & $63(74.1)$ & \multirow[t]{2}{*}{0.2} \\
\hline Non-adherent & $67(20.9)$ & $28(21.5)$ & $17(16.0)$ & $22(25.9)$ & \\
\hline \multicolumn{6}{|l|}{ Internalized stigma } \\
\hline Yes & $107(33.3)$ & $46(35.4)$ & $31(29.3)$ & $30(35.3)$ & \multirow[t]{2}{*}{0.6} \\
\hline No & $214(66.7)$ & $84(64.6)$ & $75(70.8)$ & $55(64.7)$ & \\
\hline \multicolumn{6}{|l|}{ Attitude on taking ARV } \\
\hline Positive & $278(86.6)$ & $114(87.7)$ & $90(84.9)$ & $74(87.1)$ & \multirow[t]{2}{*}{0.8} \\
\hline Negative & $43(13.4)$ & $16(12.3)$ & $16(15.1)$ & $11(12.9)$ & \\
\hline \multicolumn{6}{|c|}{ Couple HIV testing and counseling } \\
\hline Received & $129(40.2)$ & $33(25.4)$ & $60(56.6)$ & $36(42.4)$ & \multirow[t]{2}{*}{$<0.01$} \\
\hline Never received & $192(59.8)$ & $97(74.6)$ & $46(43.4)$ & $49(57.7)$ & \\
\hline \multicolumn{6}{|l|}{ Disclosed HIV status to partner } \\
\hline Disclosed & $258(80.4)$ & $109(83.9)$ & $88(83.0)$ & $61(71.8)$ & \multirow[t]{2}{*}{0.07} \\
\hline Never disclosed & $63(19.6)$ & $21(16.2)$ & $18(17.0)$ & $24(28.2)$ & \\
\hline HIV status of partner & & & & & \\
\hline Positive & $134(41.7)$ & $58(44.6)$ & $48(45.3)$ & $28(32.9)$ & 0.03 \\
\hline Negative & $52(16.2)$ & $13(10.0)$ & $23(21.7)$ & $16(18.8)$ & \\
\hline Unknown & $135(42.1)$ & $59(45.4)$ & $35(33.0)$ & $41(48.2)$ & \\
\hline
\end{tabular}


Table 1 Basic characteristics of study participants (Continued)

\begin{tabular}{lllll}
\hline Domestic violence & & & \\
Experienced & $62(19.3)$ & $28(21.5)$ & $21(19.8)$ & $13(15.3)$ \\
Not experienced & $259(80.7)$ & $102(78.5)$ & $85(80.2)$ & $72(84.7)$ \\
\hline
\end{tabular}

${ }^{\mathrm{a}} \mathrm{HC}$ : health center

bIQR: interquartile range

${ }^{\ddagger} p$-value for Chi-square test

health center was $60 \mathrm{~min}$ (IQR 30-90). About half of the women (49.5\%) were newly diagnosed as HIV-positive during the current pregnancy, and 154 women $(48.0 \%)$ were already receiving ART at enrollment to the study.

During the baseline interview, 67 women (20.9 \%) were non-adherent to ARVs. One-third (33.3\%) reported internalized stigma, and 278 (86.6 \%) showed positive attitudes towards taking ARVs. Moreover, 129 women (40.2 \%) had undergone CHTC, and 258 (80.4 \%) had disclosed their HIV status to their partners. Of all the women, $134(41.7 \%)$ reported that their partners were HIV-positive, 52 (16.2 \%) were HIV-negative, and 135 (42.1\%) had an unknown HIV status. Sixty-two women (19.3\%) reported that they had recently experienced domestic violence.

\section{Self-reported adherence to ARVs at four observation points}

Table 2 shows the proportion of adherence to ARVs among the women who made the scheduled visits (primary definition). The ARV adherence rate was $82.5 \%$ (95\% confidence interval [CI] 76.5-88.5\%) during pregnancy, $84.2 \%$ (95 \% CI 78.5-89.8 \%) at one week postpartum, $81.5 \%$ (95 \% CI 76.1-86.9 \%) at six weeks postpartum, and $70.5 \%$ (95 \% CI 62.3-78.7 \%) at 24 weeks postpartum. At the 24 weeks postpartum follow-up, five women reported cessation of breastfeeding. However, they were included in the analysis as all of them were undergoing ART.

\section{Probability of remaining adherent to ARVs}

Figure 1 shows the probability of remaining adherent to ARVs in all participants, in which loss to follow-up and infant death were treated as non-adherence (secondary

Table 2 Adherence to ARVs at four observation points ${ }^{a}$

\begin{tabular}{lllll}
\hline Observation points & Overall & Adherent & & \\
& $n$ & $n$ & $\%$ & $95 \% \mathrm{Cl}$ \\
\hline Pregnancy & 160 & 132 & 82.5 & $(76.5-88.5)$ \\
1 week postpartum & 164 & 138 & 84.2 & $(78.5-89.8)$ \\
6 weeks postpartum & 200 & 163 & 81.5 & $(76.1-86.9)$ \\
24 weeks postpartum & 122 & 86 & 70.5 & $(62.3-78.7)$ \\
\hline
\end{tabular}

${ }^{a}$ Self-reported adherence to ARVs for the four days prior to the interview (primary definition) definition). The probability of remaining adherent to ARVs was 0.61 (95 \% CI 0.56-0.66) at day 50, 0.35 (95\% CI $0.30-0.39)$ at day $100,0.18$ (95\% CI 0.14-0.21) at day 200, and 0.06 (95\% CI 0.04-0.09) at day 300.

\section{Risk factors for non-adherence to ARVs}

Before running the Cox proportional hazard models, the proportional hazard assumption was evaluated using a log-log survival plot and Schoenfeld residuals, which showed no significance in the global test $(p=0.8)$. Overall, the model appeared to meet the proportional hazard assumption. Finally, the risk factors for non-ARV adherence using the secondary definition were estimated using the Cox proportional hazard models (Table 3).

In the main final model, the women attending rural HCs providing HIV care/treatment (adjusted hazard ratio [aHR] $0.71,95 \% \mathrm{CI} 0.57-0.88$ ) and the women attending rural $\mathrm{HCs}$ not providing HIV care/treatment (aHR 0.58, 95 \% CI 0.46-0.74) were more likely to be adherent than those attending the referral HC. Women newly diagnosed as HIV-positive during pregnancy (aHR $1.24,95 \%$ CI 1.03-1.50) were more likely to be nonadherent than those with a known HIV status before pregnancy.

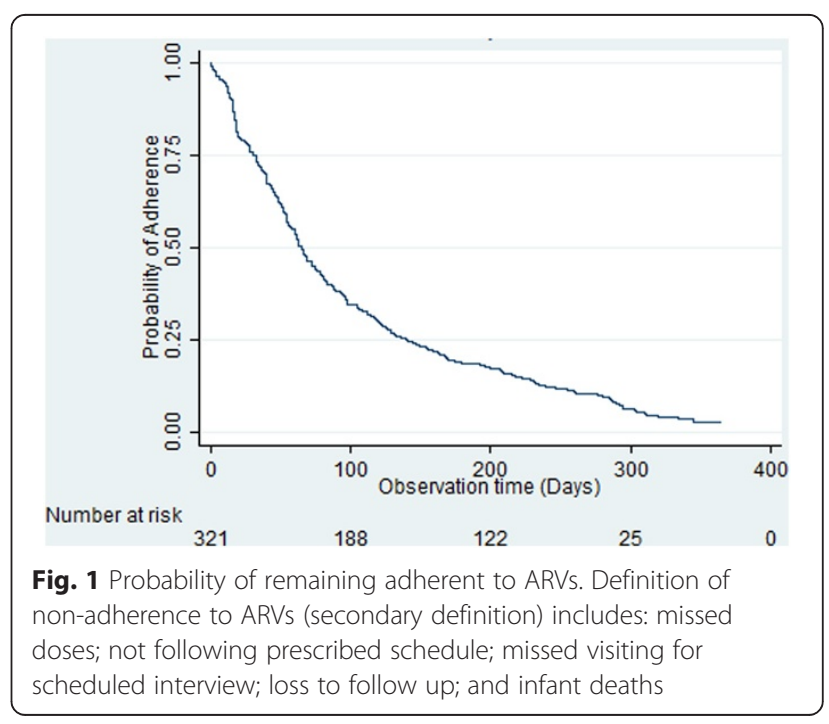


Table 3 Risk factors for non-adherence to ARV

\begin{tabular}{|c|c|c|}
\hline \multirow[t]{2}{*}{ Variables } & \multicolumn{2}{|c|}{ Main-final model } \\
\hline & $\mathrm{aHR}^{\mathrm{a}}$ & $95 \% \mathrm{Cl}$ \\
\hline \multicolumn{3}{|l|}{ Age } \\
\hline$\leq 29$ & 1.00 & \\
\hline$\geq 30$ & 0.99 & $(0.82-1.19)$ \\
\hline \multicolumn{3}{|l|}{ Educational level } \\
\hline$\leq$ Primary & 1.00 & \\
\hline$\geq$ Secondary & 0.91 & $(0.75-1.11)$ \\
\hline \multicolumn{3}{|l|}{ Marital status } \\
\hline Married/living with partner & 1.00 & \\
\hline Other & 1.03 & $(0.80-1.34)$ \\
\hline \multicolumn{3}{|l|}{ Facility type } \\
\hline Referral HC & 1.00 & \\
\hline Rural HCs with HIV care/treatment & 0.71 & $(0.57-0.88)$ \\
\hline Rural HCs without HIV care/treatment & 0.58 & $(0.46-0.74)$ \\
\hline \multicolumn{3}{|l|}{ HIV status at PMTCT enrolment } \\
\hline Already known HIV status & 1.00 & \\
\hline Newly diagnosed & 1.24 & $(1.03-1.50)$ \\
\hline
\end{tabular}

Cox proportional hazard regressions

aHR: adjusted hazard ratio

\section{Sensitivity analysis}

This study performed two sensitivity analyses to assess the following effects on the study outcome: 1) the time definition, and 2) ARV regimen. For the time definition, we utilized two models for the censorship definitions. The main model used a midpoint between the date of the previous observation and the cutoff points of the current observation, whereas the alternative model used a crude cutoff point. A comparison between the two models showed that the health facility type was a significant predictor in both models. Although the sensitivity analysis indicated that the definition of time affected the CIs of the HIV status at PMTCT enrollment, it did not show changes in the direction of the hazard ratios. Furthermore, a comparison between the two ARV regimens showed that the health facility type was a significant factor in both regimen groups, whereas the HIV status at PMTCT enrollment was a significant factor in the ART regimen group only.

\section{Mother-to-child transmission of HIV}

Table 4 shows the preliminary results of the HIV testing among infants born to the study participants. During the study period, the participants had delivered 326 infants, including five sets of twins. Although the breastfeeding status could not be confirmed, 262 infants (80.4\%) underwent HIV testing at the median age of 10.4 weeks (IQR 6.5-28.6), which was the time that they had the
Table 4 Preliminary results of the HIV testing among HIVexposed infants

\begin{tabular}{llr}
\hline HIV status & Number & Percent \\
\hline HIV positive & 11 & $(3.4)$ \\
HIV negative & 251 & $(77.0)$ \\
Unknown & 64 & (19.6) \\
\hline As of January 2014 & &
\end{tabular}

latest HIV testing, and 11 (3.4\%) were diagnosed as HIV-positive by January 2014.

\section{Discussion}

In this study, the self-reported ARV adherence rate (primary definition) was over $80 \%$ from pregnancy to six weeks postpartum, but it decreased to $70 \%$ at 24 weeks postpartum. Using the broader definition, which treated the loss to follow-up as non-adherence (secondary definition), the probability of remaining adherent to ARVs decreased considerably over the assessment period. The risk factors for non-adherence to ARVs were identified using the secondary definition, which included attending the referral $\mathrm{HC}$ and having a new diagnosis of HIV infection during the current pregnancy. Potential confounders such as the timing of study enrollment and the ARV regimen (ARV prophylaxis/ART) did not affect the study outcome. The small sample size may be one of the reasons for this finding. Eighty percent of the infants had undergone HIV testing, and $3.4 \%$ were HIV-positive, although their breastfeeding status was unknown.

This study observed poor longitudinal adherence to ARVs in the PMTCT program. Similar findings were also reported in studies conducted in other sub-Saharan African countries [5-9]. An extended ARV regimen was the major revision in the WHO 2010 guidelines [2]. The poor longitudinal ARV adherence highlights critical challenges to implementing the WHO 2010 guidelines. Another explanation for this finding would be that this study was conducted shortly after the introduction of the new guidelines; thus, the extended regimen may not have been familiar at the operational level.

In particular, women attending the referral $\mathrm{HC}$ showed poorer adherence to ARVs than those attending rural HCs. In previous studies, easier access to health facilities was found to facilitate better ARV adherence [10]. In this study, women attending the referral $\mathrm{HC}$ reported a shorter travel time to the health facility than those attending rural $\mathrm{HCs}$, which did not seem to influence their adherence. Potential reasons could be a poor quality of PMTCT services provided at the referral HC, which would be attributed to a large number of PMTCT clients per health worker. For example, only a quarter of the participants attending referral $\mathrm{HC}$ reported that they attended CHTC, whereas $>40 \%$ attended CHTC at 
other rural HCs. The gap in CHTC attendance may affect their adherence to ARVs [41-43] and the loss to follow-up [26]. A higher number of HIV patients per health workers can also increase the risk of loss to follow-up at the facility [44].

Furthermore, in rural HCs, the limited availability of laboratory and clinical services did not affect women's adherence to ARVs. This may be because the fewer number of PMTCT clients at rural HCs may allow health workers to provide effective adherence support. Similarly, a study conducted in Malawi reported that continuous follow-up by community health workers has contributed to utilization and retention in the PMTCT program [45].

Regarding the service provision system at the time of implementing this study, the women needed to be transferred to other health facilities with HIV care/treatment services. However, only $25 \%$ of health facilities offered HIV care/treatment services in Zambia in 2010 [21]. Such limited availability of the HIV care/treatment could be an obstacle for the transfer of HIV-positive women to the HIV care/treatment, which would result in loss to follow-up and treatment interruption [46]. The Zambian government has been expanding option $\mathrm{B}+$ across the country since 2013, and all target facilities of the study have introduced option B+ by August 2015. In option B +, women attending rural HCs without HIV care/treatment services will face the challenge of continuing ART even after completing the PMTCT program. To implement option $\mathrm{B}+$ across the country, effective planning is required to expand the HIV care/treatment services to all rural HCs simultaneously.

In this study, the women newly diagnosed as HIVpositive were more likely to be non-adherent than those with a known HIV status before pregnancy. A similar finding was reported in a previous study [29]. This implies that women who are newly diagnosed as HIV-positive have specific characteristics. First, the newly diagnosed women have to handle the physical and psychosocial challenges such as giving birth to a child with a risk of HIV infection, disclosing their HIV status to family members, following the daily routine of medication, and experiencing the side effects of ARVs [10, 47]. Second, they may not completely understand the importance of ARV adherence because they are more likely to be diagnosed during the asymptomatic stage; thus, they are less likely to experience the efficacy of ARV drugs $[29,48]$. Third, they may have some misconceptions or negative impressions of HIV treatment [47].

In newly diagnosed women, psychosocial support and health education would be essential interventions to overcome multiple challenges and establish good adherence to ARVs. Thus, it would be important to ensure referral of the newly diagnosed HIV-positive women to HIV care/treatment services where they are able to receive comprehensive care and support that may not be provided in the PMTCT program. However, only six facilities provided HIV care/treatment services at the study site, which is common in other parts of Zambia. This suggests that further effort is required to strengthen the linkage between the PMTCT and HIV care/treatment programs [46].

To mitigate psychosocial challenges following antenatal HIV diagnosis, women should be encouraged to undergo HIV testing before pregnancy. This will reduce the number of pregnant women who are not aware of their HIV status until the first antenatal visit. Furthermore, early awareness of their HIV status would encourage women to abstain from risky sexual behavior, which would reduce the risk of HIV transmission to their children and partners [49]. Moreover, this approach would provide the benefit of primary HIV prevention among the population of reproductive age. In this study, however, half of the participants did not know their HIV status, and $40 \%$ did not know their partners' HIV status. Similarly, nearly $70 \%$ of HIV-positive men and $50 \%$ of HIV-positive women in Zambia are reported to have never been tested before their first HIV-positive diagnosis [32]. Thus, the promotion of regular testing of all sexually active women of reproductive age would increase the benefits associated with PMTCT strategies.

This study has several limitations. First, the small sample size may limit the statistical power and affect the study findings, and it may affect the generalizability of our findings. Second, variation in the timing of study enrollment may cause selection bias even though we statistically adjusted and confirmed that the study outcome was not affected by the timing of study enrollment. Third, this study used a single measurement of self-reported ARV adherence, which was potentially affected by recall bias and social desirability bias. Forth, this study may have overestimated the incidence of non-adherence due to use of a broad definition of ARV adherence. However, this is an operational research using intention-to-treat analysis, which was designed to observe the standard practice of the PMTCT program. Finally, given the observational nature of this analysis, the results may be biased owing to the unmeasured confounders at the individual, facility, or community level.

\section{Conclusions}

The PMTCT program in Zambia is facing a crucial operational challenge of poor adherence to ARVs over the high-risk period of mother-to-child transmission. All health facilities should strengthen the support for ARV adherence and retention in care from pregnancy to the cessation of breastfeeding. 


\section{Abbreviations}

HIV: Human immunodeficiency virus; WHO: World Health Organization; PMTCT: Prevention of mother-to-child transmission of HIV; ARV: Antiretroviral; AZT: Zidovudine; NVP: Nevirapine; ART: Antiretroviral therapy; HC: Health center; CHTC: Couple HIV testing and counseling; IQR: Interquartile range; aHR: Adjusted hazard ratio.

\section{Competing interests}

The authors declare that they have no competing interests.

\section{Authors' contributions}

SO conducted the study design and analysis, supervised data collection, and drafted the manuscript. MC participated in the study design, supervised data collection, and drafted the manuscript. NI conducted the study design, supervised data collection, and drafted the manuscript. HK participated in the study design and supervised data collection. CYM, GS, SM, and KK supervised data collection. MJ and JY supervised the study design and data analysis, and helped to draft the manuscript. All authors reviewed and approved the final manuscript.

\section{Acknowledgements}

The authors would like to acknowledge the Scaling up of Quality HIV/AIDS Care Service Management Project (SHIMA Project), Zambia Ministry of Health, Japan International Cooperation Agency, and National Center for Global Health and Medicine for all their support provided to facilitate this study. We would like to express our great appreciation to Assistant Professor Stuart Gilmour and Professor Kenji Shibuya at Department of Global Health Policy, Graduate School of Medicine, The University of Tokyo, and Dr. Shinya Tsuzuki, Mr. Paul Kalichini, and Mr. Seishi Kobayashi for their technical support.

\section{Author details}

'Department of Community and Global Health, Graduate School of Medicine, The University of Tokyo, Tokyo, Japan. ${ }^{2}$ Chongwe District Community Health Office, Chongwe, Zambia. ${ }^{3}$ Ministry of Health Zambia-Japan International Cooperation Agency SHIMA project, Lusaka, Zambia. ${ }^{4}$ National Center for Global Health and Medicine, 1-21-1 Toyama, Shinjuku-ku, Tokyo 162-8655, Japan. ${ }^{5}$ Ministry of Health, Lusaka, Zambia.

\section{Received: 29 January 2015 Accepted: 6 October 2015}

\section{Published online: 12 October 2015}

\section{References}

1. UNAIDS. The gap report. Geneva: UNAIDS; 2014.

2. WHO. Antiretroviral drugs for treating pregnant women and preventing HIV infection in infants: recommendations for a public health approach: 2010 version. Geneva: WHO; 2010 [http://whqlibdoc.who.int/publications/2010/ 9789241599818_eng.pdf].

3. WHO. Consolidated guidelines on the use of antiretroviral drugs for treating and preventing HIV infection: recommendations for a public health approach June 2013. Geneva: WHO; 2013.

4. Bartlett JA. Addressing the challenges of adherence. JAIDS. 2002;29:S2-10.

5. Nachega JB, Uthman OA, Anderson J, Peltzer K, Wampold S, Cotton MF, et al. Adherence to antiretroviral therapy during and after pregnancy in low-income, middle-income, and high-income countries: a systematic review and meta-analysis. AIDS. 2012;26(16):2039-52.

6. Kirsten I, Sewangi J, Kunz A, Dugange F, Ziske J, Jordan-Harder B, et al. Adherence to combination prophylaxis for prevention of mother-to-childtransmission of HIV in Tanzania. PLoS One. 2011;6:e21020.

7. Peltzer K, Sikwane E, Majaja M. Factors associated with short-course antiretroviral prophylaxis (dual therapy) adherence for PMTCT in Nkangala district, South Africa. Acta Paediatr. 2011;100:1253-7.

8. Mepham S, Zondi Z, Mbuyazi A, Mkhwanazi N, Newell ML. Challenges in PMTCT antiretroviral adherence in northern KwaZulu-Natal, South Africa. AIDS Care. 2011;23(6):741-7.

9. Ekama S, Herbertson E, Addeh E, Gab-Okafor C, Onwujekwe D, Tayo F, et al. Pattern and determinants of antiretroviral drug adherence among Nigerian pregnant women. Journal of Pregnancy. 2012;2012:851810.

10. Gourlay A, Birdthistle I, Mburu G, lorpenda K, Wringe A. Barriers and facilitating factors to the uptake of antiretroviral drugs for prevention of mother-to-child transmission of HIV in sub-Saharan Africa: a systematic review. J Int AIDS Soc. 2013;16(1):18588.

11. Colombini M, Stockl H, Watts C, Zimmerman C, Agamasu E, Mayhew SH. Factors affecting adherence to short-course ARV prophylaxis for preventing mother-to-child transmission of HIV in sub-Saharan Africa: a review and lessons for future elimination. AIDS Care. 2014;26(7):914-26.

12. Kamuyango AA, Hirschhorn LR, Wang W, Jansen P, Hoffman RM. One-year outcomes of women started on antiretroviral therapy during pregnancy before and after the implementation of Option B+ in Malawi: a retrospective chart review. World J AIDS. 2014;4(3):332-7.

13. Ebuy $\mathrm{H}$, Yebyo $\mathrm{H}$, Alemayehu M. Level of adherence and predictors of adherence to the Option B+ PMTCT programme in Tigray, northern Ethiopia. Int J Infect Dis. 2015;33:123-9.

14. Mirkuzie AH, Hinderaker SG, Sisay MM, Moland KM, Morkve O. Current status of medication adherence and infant follow up in the prevention of mother to child HIV transmission programme in Addis Ababa: a cohort study. J Int AIDS Soc. 2011;14:50.

15. Blacher RJ, Muiruri P, Njobvu L, Mutsotso W, Potter D, Ong'ech J, et al. How late is too late? Timeliness to scheduled visits as an antiretroviral therapy adherence measure in Nairobi, Kenya and Lusaka, Zambia. AIDS Care. 2010;22:1323-31.

16. Manzi M, Zachariah R, Teck R, Buhendwa L, Kazima J, Bakali E, et al. High acceptability of voluntary counselling and HIV-testing but unacceptable loss to follow up in a prevention of mother-to-child HIV transmission programme in rural Malawi: scaling-up requires a different way of acting. Trop Med Int Health. 2005;10:1242-50.

17. Shargie MB, Eek F, Abaychew A. Prophylactic treatment uptake and compliance with recommended follow up among HIV exposed infants: a retrospective study in Addis Ababa, Ethiopia. BMC Res Notes. 2011;4:563.

18. Chetty T, Knight S, Giddy J, Crankshaw TL, Butler LM, Newell ML. A retrospective study of Human Immunodeficiency Virus transmission, mortality and loss to follow-up among infants in the first 18 months of life in a prevention of mother-to-child transmission programme in an urban hospital in KwaZulu-Natal, South Africa. BMC Pediatr. 2012;12:146.

19. Ministry of Health, National AIDS Council Zambia. Zambia country report: monitoring the declaration of commitment on HIV and AIDS and the Universal Access: biennial report. Lusaka: Ministry of Health; 2014 [http://www.unaids.org/sites/default/files/country/documents/ ZMB_narrative_report_2014.pdf].

20. Ministry of Health Zambia. 2010 National protocol guidelines: integrated prevention of mother-to-child transmission of HIV. Lusaka: Ministry of Health Zambia; 2010.

21. Ministry of Health, National AIDS Council Zambia. Zambia country report: monitoring the declaration of commitment on HIV and AIDS and the Universal Access: biennial report. Lusaka: Ministry of Health Zambia; 2012 [http://www .unaids.org/sites/default/files/en/dataanalysis/knowyourresponse/ countryprogressreports/2012countries/ce_ZM_Narrative_Report.pdf].

22. Stringer JS, Sinkala M, Stout JP, Goldenberg RL, Acosta EP, Chapman V, et al. Comparison of two strategies for administering nevirapine to prevent perinatal HIV transmission in high-prevalence, resource-poor settings. JAIDS. 2003;32(5):506-13.

23. Stringer JSA, Sinkala M, Maclean CC, Levy J, Kankasa C, DeGroot A, et al. Effectiveness of a city-wide program to prevent mother-to-child HIV transmission in Lusaka, Zambia. AIDS. 2005;19(12):1309-15.

24. Albrecht S, Semrau K, Kasonde P, Sinkala M, Kankasa C, Vwalika C, et al. Predictors of nonadherence to single-dose nevirapine therapy for the prevention of mother-to-child HIV transmission. JAIDS. 2006;41:114-8.

25. Chi BH, Chintu N, Cantrell RA, Kankasa C, Kruse G, Mbewe F, et al. Addition of single-dose tenofovir and emtricitabine to intrapartum nevirapine to reduce perinatal HIV transmission. JAIDS. 2008;48:220-3.

26. Conkling M, Shutes EL, Karita E, Chomba E, Tichacek A, Sinkala M, et al. Couples' voluntary counselling and testing and nevirapine use in antenata clinics in two African capitals: a prospective cohort study. J Int AIDS Soc. 2010;13:10.

27. Megazzini KM, Sinkala M, Vermund SH, Redden DT, Krebs DW, Acosta $E P$, et al. A cluster-randomized trial of enhanced labor ward-based PMTCT services to increase nevirapine coverage in Lusaka, Zambia. AIDS. 2010;24:447-55

28. Stringer EM, Ekouevi DK, Coetzee D, Tih PM, Creek TL, Stinson K, et al. Coverage of nevirapine-based services to prevent mother-to-child HIV transmission in 4 African countries. JAMA. 2010;304(3):293-302. 
29. Laine C, Newschaffer CJ, Zhang DZ, Cosler L, Hauck WW, Turner BJ. Adherence to antiretroviral therapy by pregnant women infected with human immunodeficiency virus: a pharmacy claims-based analysis. Obstet Gynecol. 2000;95(2):167-73.

30. Mandala J, Torpey K, Kasonde P, Kabaso M, Dirks R, Suzuki C, et al. Prevention of mother-to-child transmission of HIV in Zambia: implementing efficacious ARV regimens in primary health centers. BMC Public Health. 2009:9:314.

31. Obermeyer CM, Bott S, Carrieri P, Parsons M, Pulerwitz J, Rutenberg N, et al. HIV testing, treatment and prevention: generic tools for operational research. Geneva: WHO; 2009 [http://www.who.int/hiv/pub/ operational/or_generic_tools.pdf].

32. Central Statistical Office, Ministry of Health, Tropical Disease Research Center, University of Zambia, Macro International Inc. Zambia Demographic and Health Survey 2007. Maryland: Central Statistical Office and Macro International Inc; 2009 [http://www.measuredhs.com/ pubs/pdf/FR211/FR211[revised-05-12-2009].pdf]

33. Adult AIDS Clinical Trials Group. ACTG Self report-III. [https://www.fstrf.org/ apps/cfmx/apps/common/QOLAdherenceForms/resources/actg/forms/ english/ql0756_000_eng_v1_20091217.pdf]

34. Adult AIDS Clinical Trials Group. Supplemental antepartum adherence questionnaire. [https://www.fstrf.org/apps/cfmx/apps/common/ QOLAdherenceForms/resources/actg/forms/english/qlw0044.pdf]

35. Semrau K, Kuhn L, Vwalika C, Kasonde P, Sinkala M, Kankasa C, et al. Women in couples antenatal HIV counseling and testing are not more likely to report adverse social events. AIDS. 2005;19:603-9.

36. Chesney MA, Ickovics JR, Chambers DB, Gifford AL, Neidig J, Zwickl B, et al. Self-reported adherence to antiretroviral medications among participants in HIV clinical trials: the AACTG adherence instruments. Patient Care Committee \& Adherence Working Group of the Outcomes Committee of the Adult AIDS Clinical Trials Group (AACTG). AIDS Care. 2000;12:255-66.

37. Oyugi JH, Byakika-Tusiime J, Charlebois ED, Kityo C, Mugerwa R, Mugyenyi $\mathrm{P}$, et al. Multiple validated measures of adherence indicate high levels of adherence to generic HIV antiretroviral therapy in a resource-limited setting. JAIDS. 2004;36(5):1100-2.

38. Bardeguez AD, Lindsey JC, Shannon M, Tuomala RE, Cohn SE, Smith E, et al. Adherence to antiretrovirals among US women during and after pregnancy. JAIDS. 2008;48(4):408-17.

39. Hosmer DW, Lemeshow S, May S. Applied survival analysis: regression modeling of time-to-event data. 2nd ed. New Jersey: John Wiley \& Sons, Inc; 2008.

40. van Beek I, Dwyer R, Dore GJ, Luo K, Kaldor JM. Infection with HIV and hepatitis $C$ virus among injecting drug users in a prevention setting: retrospective cohort study. BMJ. 1998;317(7156):433-7.

41. Becker S, Mlay R, Schwandt HM, Lyamuya E. Comparing couples' and individual voluntary counseling and testing for HIV at antenatal clinics in Tanzania: a randomized trial. AIDS Behav. 2010;14(3):558-66.

42. Farquhar C, Kiarie JN, Richardson BA, Kabura MN, John FN, Nduati RW, et al. Antenatal couple counseling increases uptake of interventions to prevent HIV-1 transmission. JAIDS. 2004;37:1620-6.

43. Msuya SE, Mbizvo EM, Hussain A, Uriyo J, Sam NE, Stray-Pedersen B. Low male partner participation in antenatal HIV counselling and testing in northern Tanzania: implications for preventive programs. AIDS Care. 2008;20(6):700-9.

44. Vella V, Govender T, Dlamini S, Taylor M, Moodley I, David V, et al. Retrospective study on the critical factors for retaining patients on antiretroviral therapy in KwaZulu-Natal, South Africa. JAIDS. 2010;55:109-16.

45. Kim MH, Ahmed S, Buck WC, Preidis GA, Hosseinipour MC, Bhalakia A, et al. The Tingathe programme: a pilot intervention using community health workers to create a continuum of care in the prevention of mother to child transmission of HIV (PMTCT) cascade of services in Malawi. J Int AIDS Soc. 2012;15 Suppl 2:17389.

46. Okawa S, Chirwa M, Ishikawa N, Pande F, Kapyata H, Msiska C, et al. Operational challenge: Linkages from prevention of mother-to-child transmission services to care and treatment services in Zambia. Melbourne: Proceedings of 20th International AIDS Conference; 2014.

47. Murray LK, Semrau K, McCurley E, Thea DM, Scott N, Mwiya M, et al. Barriers to acceptance and adherence of antiretroviral therapy in urban Zambian women: a qualitative study. AIDS Care. 2009;21:78-86.
48. Barigye $H$, Levin J, Maher D, Tindiwegi G, Atuhumuza E, Nakibinge S, et al. Operational evaluation of a service for prevention of mother-tochild transmission of HIV in rural Uganda: barriers to uptake of singledose nevirapine and the role of birth reporting. Trop Med Int Health. 2010;15:1163-71.

49. Bunnell R, Opio A, Musinguzi J, Kirungi W, Ekwaru P, Mishra V, et al. HIV transmission risk behavior among HIV-infected adults in Uganda: results of a nationally representative survey. AIDS. 2008;22(5):617-24.

\section{Submit your next manuscript to BioMed Central and take full advantage of:}

- Convenient online submission

- Thorough peer review

- No space constraints or color figure charges

- Immediate publication on acceptance

- Inclusion in PubMed, CAS, Scopus and Google Scholar

- Research which is freely available for redistribution 